\title{
CYCLICAL ACTIVITY IN ENDOCRINE SYSTEMS
}

\begin{abstract}
COMPARED with members of most learned bodies, 4 Fellows of the Zoological Society of London have always been well served-more so than many have realized -in regard to the collections, the library, and the gastronomic and other facilities that they are privileged to enjoy. Additionally, in recent months, there has begun a most welcome series of one-day symposia which will in future take the place of a proportion of the ordinary scientific meetings of the Society. These symposia are not too narrowly specialist, and they are open to all zoologists whether Fellows of the Society or not.
\end{abstract}

The second such symposium, on "Cyclical Activity in Endocrine Systems", was convened by Prof. E. J. W. Barrington, and held on December 8.

The first two papers dealt with the cyclical activity of the fish thyroid. Dr. A. J. Matty (Nottingham) pointed out that although recently a number of reports have indicated a seasonal change in the thyroid, most have been derived from histological data. Only in one species has hormonal iodine been determined. Variation from species to species both in degree and time of thyroid activity made fow generalizations possible, although it is possible in some instances to correlate a seasonal thyroid change with locomotory and migratory changes. Some fish appear to have a more active thyroid during spawning, and Dr. Matty suggested that, as the gland does not seem directly to stimulate gametogenesis, the relationship is due to the influence of the thyroid on protein metabolism, for example, on gonad growth or the development of viviparous young. The thyroid in fish has been shown to influence growth, metamorphosis and integument development and, as current researches have demonstrated that nitrogen metabolism is influenced by thyroxine, it was suggested that a principal role of the piscean thyroid is the control of protein and nucleoprotein metabolism.

Dr. D. R. Swift (Windermere) made a general survey of the external factors which could affect the thyroid and its demonstrated cyclical activity. He emphasized that we do not know what the epithelial cell height and uptake and loss of radioiodine mean in terms of actual activity of the gland and that it was difficult therefore to compare the results obtained by workers using different methods. Dr. Swift suggested that the thyroid undergoes two periods of high activity, one period coinciding with the maturation of the gonads, with which it may be in fact concerned. The second period of high activity occurs as part of what is called the basic cycle of thyroid activity in these animals. This activity of the gland appears to be inversely proportional to the water temperature. It was suggested that temperature plays a major part in the control of the thyroid, but that the length of the photoperiod may influence the gland at low temperatures. It was stressed that it is the change in the environment which is important. Obviously, in Nature, the fish never experience a constant one.

Dr. P. G. W. J. van Oordt, a welcome visitor from Utrecht, is primarily interested in the Amphibia. He spoke of the influence of internal and external factors in the regulation of the anuran spermatogenetic cycle. $\mathrm{He}$ concluded that spermatogenesis depends on two so-called final factors, first (as would be expected) the gonadotrophic activity of the pituitary, and secondly, the sensitivity of the germinal epithelium to gonadotrophic hormone. Each such factor is influenced by one or more 'intermediate' factors. These include, for example, a gonadotrophin secretion-inducing agent produced by the preoptic nucleus of the hypothalamus. The male sex hormone is also influential. In Amphibia with a potentially continuous spermatogenetic cycle this activity is primarily regulated by the external temperature. This basic factor, together with the internal rhythm, determines the seasonal changes in the spermatogenetic activity of Anura with a discontinuous spermatogenetic cycle sensu stricto.

Dr. A. J. Marshall (London) next discussed cyclical phenomena and corresponding behavioural adapt. ations in relation to the environment in birds. While paying due regard to the importance of day-length changes as a regulatory factor, he remarked that if we viewed the avifauna of the world, light is important only in species for which it is important that it should be important. A whole range of widely unrelated species would be extinguished by natural forces if they were compelled to reproduce at any particular phase of the cycle of the Sun. In perennially lush areas, it is often biologically beneficialand possible-for species to have more than one breeding season per year. There is a fascinating, as yet unwritten, story of the changes in behaviour that have gone hand in hand with the neural and endocrine changes dictated by the environment and natural selection.

The complexity of endocrine cycles in vertebrates was emphasized by Prof. E. J. W. Barrington, who gave illustrations from the work of his Nottingham group to show the difficulties encountered in attempting to demonstrate a one-to-one relationship between a particular cycle and a particular function. He concluded that an essential part of the study of such cycles must be the means by which they are coordinated and brought into relationship with the external environment. This involves, in particular, a study of the pituitary and its relationships with the central nervous system. The evolution of the trophic control exerted by the adenohypophysis is one aspect of such a study, and he discussed from this point of view the relationship which has recently been claimed to exist between the adenohypophysis and the endostyle in the ammocoete larva. Results obtained at Nottingham suggest that it is by no means clear that such a relationship is demonstrable on the present evidence. Finally, he considered the problem of the relationship between the hypothalamus and adenohypophysis in teleosts and gave evidence suggesting that (contrary to what has previously been supposed) the vascularization of a particular area of the floor of the infundibulum has at least some of the characteristics of a median eminence, and that glial secretory cells are found in the same area. Identifications of such areas in other fish, and a study of any cyclical variations which 
they may show, should shed much light on the regulation of endocrine cycles in the lower vertebrates.

Dr. G. W. Harris (London), who thoughtfully made it a condition of his appearance that he would not have to write a paper, dealt comprehensively with comparative aspects of the functional relationships of the hypothalamus and adenohypophysis. He paid a graceful tribute to the pioneer work of the late F. H. A. Marshall in the field of reproduction physiology.

The final two papers were concerned with invertebrates. Dr. M. J. Wells (Cambridge) gave an account of some of his work on the optic glands and the ovary of Octopus and described a hormonal system which regulates the onset of sexual maturity. In cephalopods, maturation of the gonad depends upon release of a protein secretion from the optic glands. These are in turn governed by an inhibitory nerve supply originating in the highest centres of the central nervous system. The optic glands can be activated (causing precocious sexual maturity) by severance of this nerve supply, or by peripheral blinding. 'Ripening' of the oviducts and oviducal glands is independent of ovarian maturation. The system is single-stage; the state of these secondary structures is not under the control of sex hormones. The brain-optic gland system of cephalopods has obvious analogues in chordates and arthropods; the factors determining the convergent evolution of mechanisms for delaying the onset of sexual maturity were discussed.

The final paper was by Dr. D. B. Carlisle (Plymouth) on moulting cycles in Crustacea. The act of moulting in arthropods, said Dr. Carlisle, must not be considered as an isolated event but as the most spectacular moment in a continuous cycle of metabolic activity. Each moulting cycle is basically like the one before it, and many of the stages have now been shown to be under hormonal control. Secretion of the 'moult inhibiting hormone' by the endocrine complex of the eye-stalk promotes the formation of new tissues and prevents the $Y$ organ from secreting more than 'marginal' amounts of its hormone. Once this eye-stalk hormone ceases to be secreted the $Y$ organ releases a single 'dose' of a moult-promoting hormone. This initiates the preparations for the moult which, once under way, are co-ordinated by a moult-accelerating hormone from the $X$ organ. The amount of water taken in at the moult itself is regulated by a further eye-stalk hormone. Many other hormones act upon particular moults and modify them in some way, acting mainly upon the stage of proecdysis and making the succeeding moult in some way unique : the moult of puberty and the moult at which a female becomes furnished with the brooding characters are examples. In the same way that the originally similar segments of the vertebrate body have been progressively and uniquely modified (in, for example, the head) so the originally similar moults of arthropods have been modified, until we have what Dr. Carlisle called 'the startling difference' between the larval and imaginal moults of Lepid. optera.

Prof. J. Z. Young, who was chairman of the invertebrate session, enlarged upon a statement made by the final speaker concerning the complications of modern endocrinology. Prof. Young said that the presence of a multiplicity of hormones was the natural consequence of the fact that they were sent out, broadcast through the body and each needed to be addressed to a particular target. No such multiplicity of chemical mediators was necessary in the nervous system, where addresses were specified by connexions. The discovery of more and more distinct hormones was a sign of the maturity of the subject. Endocrinologists were learning to understand the code with which their complicated system operated.

The symposium concluded with a general discussion and a summary by Sir Solly Zuckerman. The papers will be published soon as one of the Symposia of the Zoological Society of London. A. J. MARSHALL

\section{AERODYNAMIC CAPTURE OF PARTICLES}

A SYMPOSIUM on this subject was held during January 13-14 in the laboratories of the British Coal Utilization Research Association at Leatherhead.

Although this might be thought to be a field of very limited appeal, yet, as evidenced by the wide interests of the fifty scientists present, the impingement of small partieles carried in a stream of air and falling on to a solid surface placed alongside or in the way of the flow is a problem which concerns a number of industries, as well as having meteorological implications ranging from the ieing of aireraft wings and motor-car windscreens to defence against microbiological warfare.

The wide scope of the applications of this study can be seen by looking through the list of titles offered at this symposium. In 'pure' meteorology one studies cloud formation and collision of raindrops, in applied meteorology the wash-out by rain of particulate matter, whether this be the product of industrial air pollution or of an atomic explosion. A number of cognate problems arise in the laying of mine dusts by water sprays. Then we have the impact of water drops or ice crystals on large moving surfaces such as aircraft wings or turbine blades; when both the obstacle and particles are on a smaller scale we have the action of the gas-mask filter (one of the earliest applications).

The coagulation of particles carried by a stream of air or water is, of course, of great interest and involves many applications where solid or liquid particles have to be transported from place to place, for example, in the industrial process of fluidization.

The basic theory of much of this work stems from Albrecht, who worked out in 1931 the proportion of particles carried by a stream of aix which would be intercepted by a cylinder placed athwart the stream. The particles tend to follow the stream lines as they approach the cylinder, but their mass makes them continue in a forthright direction. If their resistance in the stream is given by Stokes's law, that is, direct proportionality to velocity and viscosity, their actual paths depend on this balance between inertia and viscosity in a parameter resembling a particle Reynolds number. This 'particle parameter' has been 